\title{
THE ARENS IRREGULARITY OF AN EXTREMAL ALGEBRA
}

\author{
by M. J. CRABB and C. M. MCGREGOR
}

(Received 25th August 1992)

A class of extremal Banach algebras has Arens irregular multiplication.

1991 Mathematics subject classification (1985 Revision): 46J35.

For any compact convex set $K \subset \mathbb{C}$ there is a unital Banach algebra $E a(K)$ generated by an element $h$ in which every polynomial in $h$ attains its maximum norm over all Banach algebras subject to its numerical range $V(h)$ being contained in $K,[1,2]$. In [3] we showed that $E a(K)$ does not have Arens regular multiplication when $K$ is a line segment. Here we extend this to any other case, where a different argument is required.

Proposition. If $K$ has non-empty interior, then $E a(K)$ is Arens irregular.

Proof. We use Pym's criterion [4] that it is enough to find bounded sequences $a_{n}, b_{n}$ in $E a(K)$ and a bounded linear functional $\phi$ such that the two repeated limits of $\phi\left(a_{m} b_{n}\right)$ exist and differ.

First assume that $K=\bar{D}(0, \tau)$, where $\tau=4 / e$. As in [2], any entire function $f$ such that $f(z) e^{-r|z|}$ is bounded on $\mathbb{C}$ gives a $\phi$ in $E a(K)^{\prime}$ by $\phi\left(e^{z h}\right)=f(z)(z \in \mathbb{C})$. Define an entire function $f$ by

$$
f(z)=4 z^{2} \prod_{p=1}^{\infty}\left[1-\left(\frac{z}{2^{p}}\right)^{2 p}\right]
$$

and put

$$
g_{n}(z)=4 z^{2} \prod_{p=1}^{n}\left(\frac{z}{2^{p}}\right)^{2 p}=\left(\frac{z}{2^{n-1}}\right)^{2^{n+1}} \quad(n \in \mathbb{N})
$$

Then 


$$
\begin{aligned}
(-1)^{n} \frac{f(z)}{g_{n}(z)}= & \prod_{1}^{n-1}\left[1-\left(\frac{2^{p}}{z}\right)^{2 P}\right] \cdot\left(1-\left(\frac{2^{n}}{z}\right)^{2^{n}}\right) \\
& \times\left(1-\left(\frac{z}{2^{n+1}}\right)^{2^{n+1}}\right) \cdot \prod_{n+2}^{\infty}\left[1-\left(\frac{z}{2^{p}}\right)^{2 P}\right] \\
= & A_{n}(z) B_{n}(z) C_{n}(z), \text { say. }
\end{aligned}
$$

Put $D_{n}=\left\{z \in \mathbb{C}: 2^{n} \leqq|z| \leqq 2^{n+1}\right\}$. Then $A_{n}(z) \rightarrow 1$ as $n \rightarrow \infty$ uniformly on $D_{n}$, since

$$
\sum_{1}^{n-1}\left|\frac{2^{p}}{z}\right|^{2^{p}} \leqq \sum_{1}^{n-1} 2^{(p-n) 2^{p}} \leqq(n-1) 2^{2(1-n)} \rightarrow 0 .
$$

Further, $C_{n}(z) \rightarrow 1$ uniformly on $D_{n}$, since

$$
\sum_{n+2}^{\infty} 2^{(n+1-p) 2^{p}} \rightarrow 0
$$

Since also $\left|B_{n}(z)\right| \leqq 4\left(z \in D_{n}\right)$, we have $\left|f / g_{n}\right|<5$ on $D_{n}$ for all large enough $n$. Together with the fact that $g_{n}(r) e^{-\tau r} \leqq 1(r>0)$, this gives that $f(z) e^{-\tau|z|}$ is bounded on $\mathbb{C}$. Thus $f$ defines $\phi$ in $E a(K)^{\prime}$ as described.

With some fixed $\alpha \in \mathbb{R}$, put $r_{n}=2^{n-1} e+\alpha(n \in \mathbb{N})$. Then $r_{n} \in D_{n}$ for all large $n, B_{n}\left(r_{n}\right) \rightarrow 1$, and (1) gives that $(-1)^{n} f\left(r_{n}\right) / g_{n}\left(r_{n}\right) \rightarrow 1$.

Also, $\log \left[g_{n}\left(r_{n}\right) e^{-\tau r_{n}}\right]=2^{n+1} \log \left(e+2^{1-n} \alpha\right)-4 r_{n} / e=2^{n+1} \log \left(1+2^{1-n} \alpha / e\right)-4 \alpha / e \rightarrow 0$ as $n \rightarrow \infty$. Hence $g_{n}\left(r_{n}\right) e^{-\tau r_{n}} \rightarrow 1$, and

$$
(-1)^{n} f\left(r_{n}\right) e^{-\tau r_{n}} \rightarrow 1 \text { as } n \rightarrow \infty .
$$

Now for $n \in \mathbb{N}$ put $\alpha_{n}=2^{2 n} e, \beta_{n}=2^{2 n-1} e$, and $a_{n}=e^{\alpha_{n}(h-\tau)}, b_{n}=e^{\beta_{n}(h-\tau)}$. Then $a_{n}$, $b_{n} \in E a(K)$ with $\left\|a_{n}\right\|=\left\|b_{n}\right\|=1$, and $\phi\left(a_{m} b_{n}\right)=f\left(\alpha_{m}+\beta_{n}\right) e^{-\tau\left(\alpha_{m}+\beta_{n}\right)}$. Since $\alpha_{m}+\beta_{n}=$ $2^{2 n-1} e+\alpha_{m}$ can be taken as $r_{2 n}$ in (2), we have $\lim _{n \rightarrow \infty} \phi\left(a_{m} b_{n}\right)=1$. With $n$ fixed, $\alpha_{m}+\beta_{n}$ can be taken as $r_{2 m+1}$, and (2) gives $\lim _{m \rightarrow \infty} \phi\left(a_{m} b_{n}\right)=-1$. Thus the repeated limits of $\phi\left(a_{m} b_{n}\right)$ differ, and $E a(K)$ is Arens irregular.

Given any compact convex $K \subset \mathbb{C}$, by replacing $K$ by $\alpha K+\beta$ for suitable $\alpha, \beta \in \mathbb{C}$ we can assume that $\bar{D}(0, \tau) \subseteq K$ and $\operatorname{Re} K \leqq \tau$. Then we can construct $\phi, a_{n}, b_{n}$ in exactly the same way to complete the proof; for since $\left\|e^{z h}\right\|=\max \left\{\left|e^{\lambda z}\right|: \lambda \in K\right\}$ we still have $\left\|a_{n}\right\|=\left\|b_{n}\right\|=1$ and $\phi \in E a(K)^{\prime}$.

\section{REFERENCES}

1. B. Bollobas, The numerical range in Banach algebras and complex functions of exponential type, Bull, London Math. Soc. 3 (1971), 27-33. 
2. M. J. Crabb, J. Duncan and C. M. McGregor, Some extremal problems in the theory of numerical ranges, Acta Math. 128 (1972), 23-42.

3. M. J. Crabb and C. M. McGregor, Interpolation and inequalities for functions of exponential type: the Arens irregularity of an extremal algebra, Glasgow Math. J., to appear.

4. J. S. Рүм, The convolution of functionals on spaces of bounded functions, Proc. London Math. Soc. (3) 15 (1965), 84-104.

Department of Mathematics

UNIVERSITY OF GLASGOW

UNIVERSITY GARDENS

GLASGOW G12 8QW 